\title{
Replacing Special Relativity
}

\author{
J.C. Hodge ${ }^{1 *}$ \\ ${ }^{1}$ Retired, 477 Mincey Rd., Franklin, NC, 28734
}

\begin{abstract}
The Special Theory of Relativity applies where gravitation is insignificant. There are many observations that remain poorly explained by the standard models of either the big of cosmology or the small of Quantum mechanics. The strength of the Scalar Theory Of Everything (STOE) is its ability to describe an extremely wide range of observations and to predict observations. Each of the STOE axioms has been used in the development of models of observations in the big and the small. The axioms that replace Special Relativity are: (1) Time is an abstraction of the duration between events causing events. (2) The diameter of the hods is the same throughout the universe. (3) The distance between hods is related to plenum density $\rho$. Higher $\rho$ reduces the distance between hods. (4) The speed of photons and hods (light) is the greatest of any matter in a given environment. (5) The speed of the plenum wave is much faster than the speed of the hods. The STOE passes the tests of Special Relativity and does much more. The STOE is a major paradigm shift.
\end{abstract}

keywords: STOE, special relativity

\section{INTRODUCTION}

The theory of Special Relativity (SR) was developed from consideration of the aberration of light, Lorentz's elaborations of Maxwell's equations (independence of the speed of light $c$ of the source and observer), the moving magnet and conductor experiment, the null results of aether drift experiments, and the Fizeau experiment (suggesting the $c$ is modified to $c / n$ where $n$ is the index of refraction). Additional experiments considered to be tests of SR are the Doppler effect, the Kennedy-Thorndike like experiments (testing constancy of $c$ ) and Ives-Stilwell like experiments (testing time dilation and length contraction).

The Scalar Theory Of Everything (STOE) was developed to model cosmological problems (Hodge 2015d, 2020). Hodge (2004) posited the universe was composed of two components and their interaction. The STOE is causative, Machian, and fractal (self-similar).

The STOE application to SR started with Newton's speculations about corpuscular nature of light (Hodge 2012, 2020). A particle model of diffraction and

${ }^{*}$ E-mail: jchodge@frontier.com 
interference must first describe "coherence" of light. Passing the light through a slit in a mask tests coherence. If the light is coherent, a diffraction pattern appears on a screen. If the light is not coherent, a diffraction pattern will not appear on a screen.

Hodge (2012) expanded on the characteristics of the plenum, hods, and their interactions to derive the STOE particle ${ }^{1}$ photon diffraction model. This photon model and a toy simulation program were developed to yield a diffraction pattern after random particle photons moved a large distance that simulated the development of coherence of light. The computer program involved several iterations, which raises the specter of chaos. However, chaos is avoided by having several feedback conditions that are also in nature. Passing the photons through a slit and matching the screen pattern to a Fraunhofer pattern demonstrated coherence. Other observations suggest the photon distribution in a laser beam and explain the Afshar Experiment (Ashar 2007).

The photon model was extended and modified to describe the single photon at a time in the experiment (Hodge 2015b,c, and references therein). This model suggested the experiments (Hodge Experiments) involving the varying illumination of coherent light across a slit(Hodge 2017b,c). The prediction was found to be consistent with the observations of Hodge Experiments. In addition, an experiment involving a transparent mask rejected wave models of light. Only the STOE model of interference was not rejected (Hodge 2019a). One of the characteristics of this model is that the $c$ varies linearly with the $\rho$ and is the highest speed that any matter (hods) may achieve. Because the $\rho$ caused by the Sun and the universe may vary locally to the photon without being in a refractive substance, this model differs from the Fizeau model.

The STOE addressed Maxwell's Equations by experiments that suggested the "moving magnet and conductor thought experiment" had a basis in a different view of the Biot-Savart Law and the magnetic field (Hodge 2018a,b,c).

This paper explores how the STOE applies to the Special Relativity basic observations. The sections of this paper discuss the aspects of Special Relativity: 2 Index of refraction

3 Null Experiments

4 Length contraction

5 Moving magnet and conductor problem

6 Clocks

7 Time dilation

8 Discussion and Conclusion.

\section{Index of refraction}

Figure 1 shows the diffraction simulation trajectories of photons (Hodge 2015a). Note the photons start to change direction toward the mask before they reach the mask. Experimenters have noted this effect. Also, the speed of light changed

\footnotetext{
${ }^{1} \mathrm{~A}$ distinction is made between a wave packet type model that is called a "photon" and a particle type model.
} 


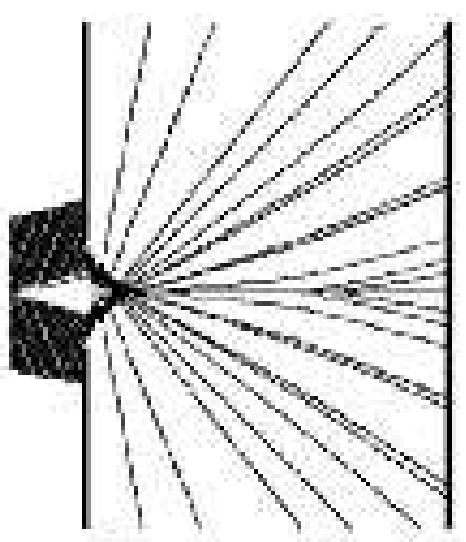

Figure 1: Photon diffraction simulation from Hodge (2015a).

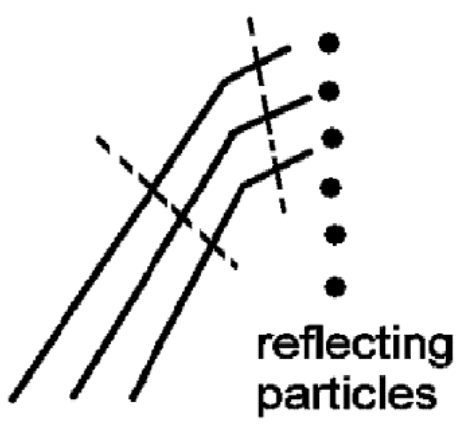

Figure 2: Index of refraction starts before photons enter the new media.

in the slit (Verdad 2019). This is consistent with the unexplained observation that the light beam changes direction immediately before entering a different medium such as from air to glass, see Fig. 2.

Light from distant objects (stars) achieves coherence. The STOE model of this was demonstrated in a computer simulation (Hodge 2012). The part of the model that produced coherence is that each particle causes a variation in the $\rho$ field. The other photons then travel faster or slower depending on the $\rho$ as depicted if Fig. 3. The Shapiro delay is interpreted to mean the lower $\rho$ closer to bodies causes a lower speed of photons. Higher $\rho$ causes faster photon speeds.

The photons/bodies in a trough also reinforce the trough for other photons. Thus, the photons tend to align in bunches which are coherent as diagramed in Figs. $4 ; 5 ; 6$; and 7 .

The model of a photon (Hodge 2012) included the simulation of how a random distribution of photons become coherent with distance traveled. The forces on the photons force them to become organized in accordance with the number of hods (frequency) in a photon as depicted in Figs. 4. The speed of photons is the fastest that matter can travel in any environment. If the source is moving away from the direction of previously emitted photons, the rate spacing of the photons becomes longer Fig. 5. The spacing of the bunches changes. The STOE suggests the forces on the photon tend toward becoming coherent. The addition of hods to matter particles has been assumed for galaxy redshift, the Doppler effect, and for the generation of the magnetic field of electromagnetic waves of photons. They become coherent by attracting free hods or by ejecting hods during their travel. The coherent grouping is reestablished (see Figs. 6 and 7).

A distinction is made between the velocity of an object such as hods that depends on the environment $(\rho$ and $n$ ) along its path and the velocity of an object such as bullets and balls that depend on the velocity of the source. 


\section{NULL EXPERIMENTS}

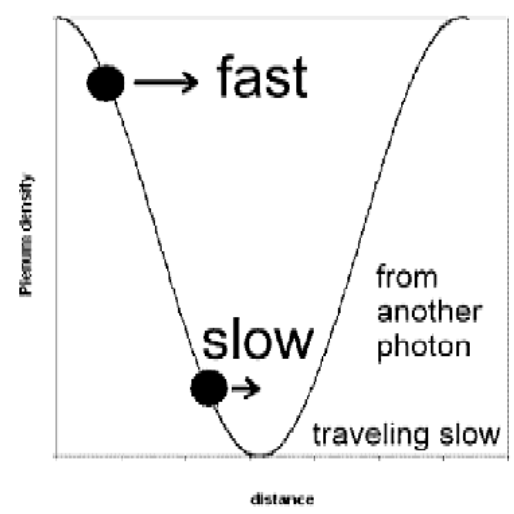

Figure 3: Photons change speed depending on $\rho$ from Hodge (2012).

The Fizeau experiment demonstrated the speed of light $c$ is higher in the direction of fluid flow and slower against the fluid flow. This is the same mechanism. That is, the immediate environment of the photon determines the photon's trajectory. This effect is called "carry-along".

Carry-along is not a drag effect. Carry-along derives from a necessary condition in the toy simulation to produce photon interference. The concept of "drag" refers to a major body such as the Earth dragging the aether to create a co-moving situation. The carry-along is a varying plenum that causes photons to change speed amongst atoms.

\section{$3 \quad$ Null experiments}

The null experiments such as the Michelson-Morley Experiment, the Miller Experiment, and similar experiment are explained because the $\rho$ and $\vec{\nabla} \rho$ (gravity) are carried-along with the experimental apparatus. The overall $\rho$ caused by the Sun and Moon cause tides (Hodge 2019b). The changes around the experiment are too slow and may have caused the 6 P.M. observations to be slightly higher than the noon observations (Sun perpendicular to the plane of the experiment) (Michelson \& Morley 1987). Therefore, there is no "wind" causing the required change in the diffraction pattern, but a tidal force of $\vec{\nabla} \rho$ is present.

Note that the theory used by Michelson-Morley is inconsistent with the Fizeau experiment.

The STOE model suggests the carry-along concept will produce a zero result. But the result was not zero and was in a different direction than a possible "wind". Perhaps Fitzgerald was correct in suggesting a real contraction of matter. The STOE suggests a contraction based on the $\rho$ value in the immediate 


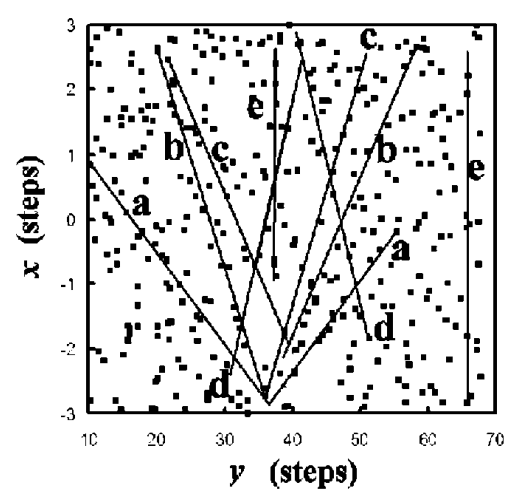

Figure 4: Coherent distribution of photons from Hodge (2012).

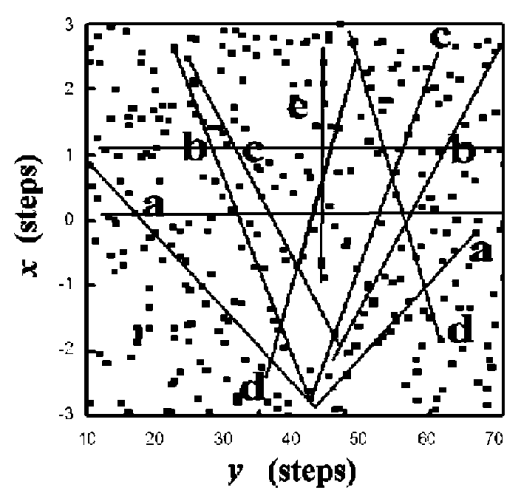

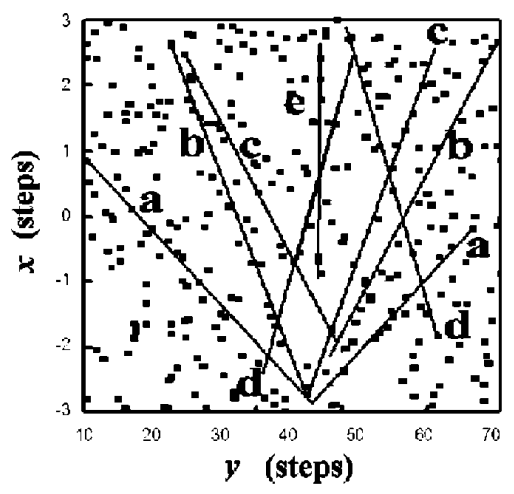

Figure 5: Distribution of coherent photons if source moving away.

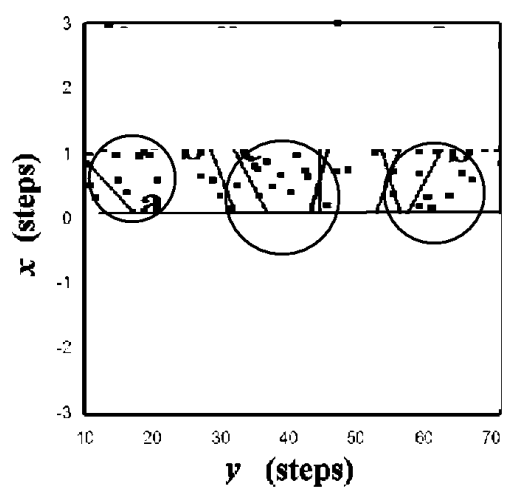

Figure 6: Distribution of coherent Figure 7: Distribution of coherent photons if source moving away fo- photons if source moving away focused view. cused view with the concentrated areas circled and after moving through a slit. 
environment of the matter such as across the experimental apparatus. This contraction is speculated to be a variation between atoms or between hods caused by the variation of the overall $\rho$ value.

The STOE speculates that bodies are composed of hods held a distance between hods. This model was simulated by disk magnets such as seen in Fig. 8 for a neutrino (Hodge 2015e). If the distance between hods is reduced such as depicted in Fig. 9, the body is subject to disintegration. This lessens the particles' size but not the gravitational mass. Thus, particles contract toward the center of spiral galaxies and become black holes. The black holes compress to release high-energy photons which radiate outward as observed in periodic Xray bursts without accompanying radiation of other frequencies (Hodge 2006b). A subset of length contraction is aberration because of the direction of photon movement.

The STOE speculation of atomic structure is that electrons are held in place by photons that are columns of hods such as depicted in Fig. 10 (Hodge 2019i). Also, such as compression may be the Heaviside Ellipsoid compression of the coulomb field. Such a structure would also compress with varying $\rho$ that would cause the distance between atoms to change as $\rho$ changes.

\section{Length contraction}

Length contraction is a measurement phenomenon. That is, the length of rods does not change from one inertial frame to another due to the relative velocity. Figure 11 shows the actual length of a rod $l=P_{3}-P_{1}$ in the comoving coordinate system where the P's are points of interest. The length $l^{\prime}=P_{2}-P_{1}$ as measured in another coordinate system $(\mathrm{O})$ moving at a constant velocity $v$. The distance moved during the time $t$ a photon takes to move from $P_{1}$ to $O$ is $v t$ as shown on the diagram. $P_{2}$ was the position of the end $\left(P_{3}\right)$ at $t=0$. During $t$, light travels toward the observer and moves a distance of $c^{\prime} t$. That is, the direction of light appears to originate from each end of the rod. Therefore, the $l^{\prime}<l$ by the Lorentz factor.

The measured length contraction depends on the speed of the communication such as light speed or sound speed. Measuring gravitational mass depends on the speed of a wave in the plenum. The speed of plenum waves is the speed of gravity which is many times the speed of light (van Flandern 1998). For example, Fig. 12 depicts the difference in identifying the center of a moving rod measured by light and by the plenum wave (gravity).

A subset of length contraction is aberration because of the direction of photon movement.

\section{Moving magnet and conductor problem}

Maxwell's Equations suggests fields that transform according to Lorentz invariance. The STOE posits hods are permanent magnets. Electromagnetic 


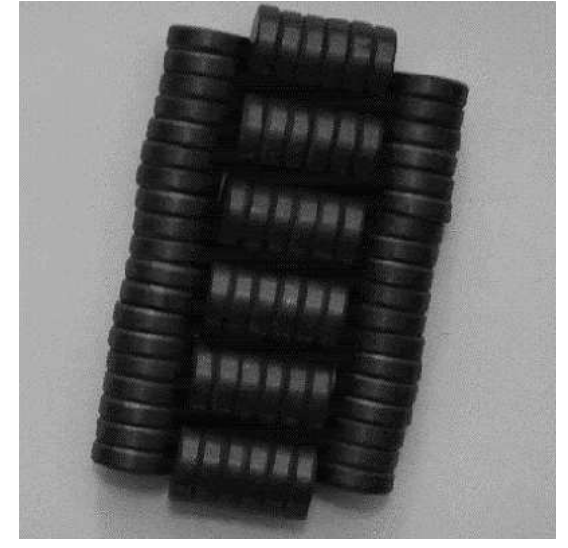

Figure 8: STOE structure of a neutrino in our region of the Milky Way Hodge (2015e).

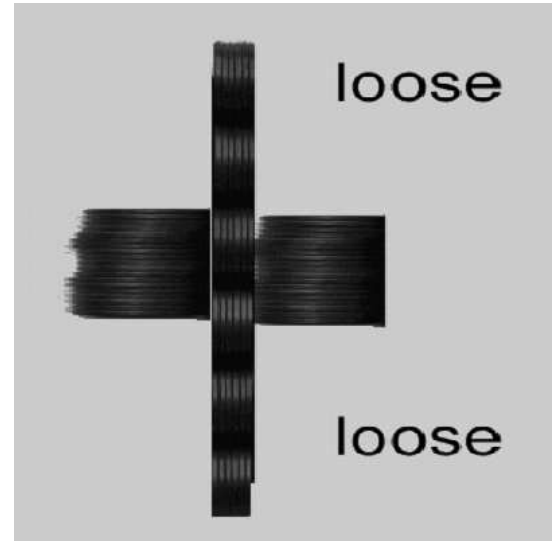

Figure 9: STOE structure of a neutrino in a region of the Milky Way closer to the center.

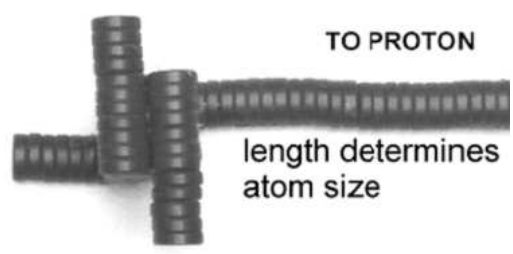

electron

Figure 10: Distribution of coherent photons if source moving away focused view (Hodge 2019i). 


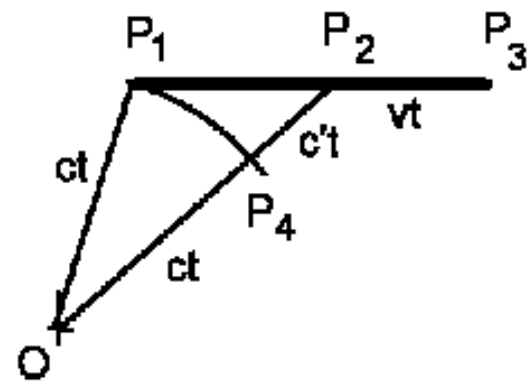

Figure 11: The length of a rod and its measured length.

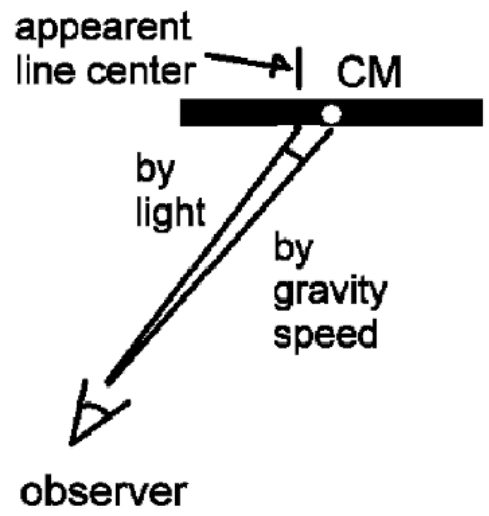

Figure 12: The length of a rod and its center as measured by light and as measured by gravity.

radiation signals are hods that travel at the speed of light. The magnetic field is the plenum density that is high on one side of a hod and zero on the other side (Hodge 2019j). The coulomb force is a plenum effect because it travels at the speed of the plenum (de Sangro 2012).

The moving magnet and conductor problem is a variant of the earlier Faraday paradox associated with Maxwell's Equations. If a magnet is in motion and a conductor is stationary in a lab frame, an electric field arises in the conductor. If the conductor is in motion and the magnet stationary, no electric field around the magnet arises.

The STOE has noted an inconsistency between Faraday's law of induction and Ampere's circuit law that derives from the Biot-Savart law. The experiment of Hodge (2018a,b) demonstrates the magnetic effect of a current in a wire is ejected perpendicular to the wire. That is, the integration along the entire length of the wire in the Biot-Savart law is incorrect. The STOE suggests the hods with their magnetic characteristic are ejected from the wire. Calculations using the Biot-Savart law's cross product or Ampere's circuit law dot product are incorrect.

Another experiment demonstrates the magnetic field induced by a electric current traveling at hod speed is not the same magnetic field from permanent magnets traveling at plenum wave speed (Hodge 2018c). That is, two different types of magnetic field that in Maxwell's Equations are considered as one variable. Therefore, the speed of an electromagnetic wave (speed of light) calculation from Maxwell's equations is incorrect.

Consider an analogy of a wave on a string,

$$
v^{2}=\frac{T}{\sigma}
$$




\section{CLOCKS}

where $v$ is the velocity of the wave, $T$ is the tension on the string, and $\sigma$ is the inertia per unit length that keeps the string moving.

The STOE suggests the hod is a permanent magnet that warps the plenum density. The warp causes gravity. The warp is a tension in the $\rho$ field. The plenum has the property of inertia and the inertia of bodies is the captured plenum in a body (Hodge 2017a). The speed limit of the hod depends on its inducing a tension in the plenum and the $\rho$ environment.

$$
\begin{gathered}
v^{2} \sim c^{2} \\
T \sim \mu^{-1} \\
\sigma \sim \epsilon \\
c^{2}=\frac{\mu^{-1}}{\epsilon},
\end{gathered}
$$

where $c$ is the maximum speed of the hod(s) or electromagnetic speed, $\mu$ is the permeability of the plenum environment, and $\epsilon$ is the permittivity of the plenum environment. The $\mu$ and the $\epsilon$ are the proportionality constants in Maxwell's Equations as re-interpreted by the STOE.

\section{Clocks}

Clocks are assumed (designed) to produce a uniform duration between ticks (events). They consist of two parts, the physical process that is repeating and the counting mechanism. Therefore, the "clock" is really just comparing one physical process with another. The "time" abstract concept is a misinterpretation in physics.

Consider a pendulum clock. The pendulum clock is well modeled. We can predict the tick rate if the clock is placed in a box and dropped. The time between ticks slows if not stops. Similarly, the tick rate slows or stops if placed in an accelerating plane, at a higher altitude, etc.

We have no model of the decay rates of atomic clocks beyond the statistical description. But, the statistical description omits the mechanism of decay. Without the knowledge of the mechanism of decay, ascribing the rate of time progression to time dilation is questionable (Rautio 2020). For example, the muon decay rate while falling may be analogous to a free falling pendulum clock in a box. The change in decay rate may indicate the cause of decay.

\section{Time dilation}

The concepts of "space" and "time" are only backdrops in the STOE where events play out their trajectories. "Space" allows movement to and back and is distance between objects. "Time" is only in one direction, the "arrow of time" and is the duration between events. Processes are NOT symmetric as current physics suggests measured by "entrophy". 


\section{DISCUSSION AND CONCLUSION}

Another phenomenon attributed to time dilation is two different photons traveling through the plenum with different $\rho$. For example, the Shapiro delay of the photons traveling closer to the Sun is traveling through a lower $\rho$ than the photon traveling farther from the Sun. The STOE suggest the time difference is because the slower speed of the photons traveling closer to the Sun. Therefore, the delay is the slower speed not time dilation. Another phenomenon is the bending of light around massive objects. The inner photons travel slower. This is consistent with the Hodge diffraction experiment (Hodge 2015c).

\section{Discussion and Conclusion}

The list of problematical observations that the STOE explains continues to grow with an improved understanding of the universe (Hodge 2015d, 2016, 2020).

The scalar $\rho$ depends on all the masses, Sources and Sinks in the universe. The $\vec{\nabla} \rho$ gives the "gravity" and Mach's Principle. The STOE Relativity considers the accelerated frame indistinguishable from the inertial frame. This has already been used to calculate the galaxy redshift and the Pioneer Anomaly. This gives the Poisson Equation. The STOE extends into General Relativity scales by considering the changes in position of the masses, Sources, and Sinks which will yield the d'Alembert's Equation without the complexity of tensor General Relativity that involves the conversion to geometry and the inverse conversion to gain physical observations.

The STOE calculates all physical quantities as invariant under velocity except as specifically calculated or as caused by changes in $\rho$. Therefore, the laws of nature are scale invariant. Therefore, the relation of gravity and scale invariance becomes clear.

The STOE rejects the notion of spacetime because of the arrow-of-time. The STOE arrives at the Equivalence Principle by a particle structure argument (Hodge 2015e). Therefore, geometric gravity and spacetime are unnecessary.

Each of the STOE axioms has been used in the development of models of observations in the big and the small. The strength of the Scalar Theory Of Everything (STOE) is its ability to describe an extremely wide range of observations and to predict observations. The axioms that replace Special Relativity are: (1) Time is an abstraction of the duration between events causing events. (2) The diameter of the hods is the same throughout the universe. (3) The distance between hods in a particle is related to plenum density $\rho$. Higher $\rho$ reduces the distance between hods. (4) The speed of photons and hods (light) is the greatest of any matter in a given environment. And (5) The speed of the plenum wave is much faster than the speed of the hods. The STOE passes the tests of Special Relativity and does much more. The STOE is a major paradigm shift.

ORCID iD

John C. Hodge: https://orcid.org/0000-0002-1520-2153 


\section{REFERENCES}

\section{References}

Afshar, S.S., 2007, Violation of the Principle of complementarity, and its implications, Proc. SPIE 5866, p. 229 http://www.arxiv:quant-ph/0701027 DOI 10.1117/12.638774

de Sangro, et al., 2012, Measuring Propagation Speed of Coulomb Fields, https://arxiv.org/abs/1211.2913 .

Hodge, J.C., 2004, Changing universe model with applications, http://www.arxiv.org/abs/astro-ph/0409765v1

Hodge, J.C., 2006a, Scalar potential model of redshift and discrete redshift, New Astronomy, Volume 11, Issue 5, March 2006, Pages 344358. http://www.sciencedirect.com/science/article/pii/S1384107605001387. http://www.arxiv.org/abs/astro-ph/0602344v1

Hodge, J.C., 2006b, Scalar potential model of the CMB radiation temperature, https://www.arxiv.org/abs/astro-ph/0603140

Hodge, J.C., 2006c, Scalar potential model of the Pioneer Anomaly, http://www.arxiv.org/abs/astro-ph/0612567v2

Hodge, J.C., 2012, Photon diffraction and interference, IntellectualArchive, Vol.1, No. 3, P. 15, http://intellectualarchive.com/?link=item\&id=597

Hodge, J.C., 2015a, Single Photon diffraction and interference, http://intellectualarchive.com/?link=item\&id=1557

Hodge, J.C., 2015b, Light diffraction experiments that confirm the STOE model and reject all other models, http://intellectualarchive.com/?link=item\&id $=1578$

Hodge, J.C., 2015c, Diffraction experiment and its STOE photon toy simulation program rejects wave models of light, http://intellectualarchive.com/?link=item\&id=1603 see video "stoe photon diffraction".

(https://www.youtube.com/channel/UCc0mfCssV32dDhDgwqLJjpw)

Hodge, J.C., 2015d, Universe according to the STOE, http://intellectualarchive.com/?link=item\&id $=1648$

Hodge, J.C., 2015e, Structure and spin of the neutrino, electron, and positron, IntellectualArchive, Vol.5, No. 2, P.1-8 , http://intellectualarchive.com/?link=item\&id $=1648$

Hodge, J.C., 2016, STOE assumptions that model particle diffraction and that replaces $Q M$, http://intellectualarchive.com/?link=item\&id=1719

Hodge, J. C., 2017a. STOE inertia, IntellectualArchive, v.6(1), P. 158,http://intellectualarchive.com/?link=item\&id=1814. 


\section{REFERENCES}

Hodge, J.C., 2017b, Hodge experiment (continued) of interference with a slit in a transparent mask rejects wave models of light, IntellectualArchive, Vol.6, No. 6, p.1-6 http://intellectualarchive.com/?link=item\&id=1862 video: https://www.youtube.com/watch?v=A07bogzzMEI

Hodge, J.C., 2017c, Hodge experiment (continued) with opaque strips and about the Afshar Experiment, IntellectualArchive, Vol.6, No. 5, p.7-18 http://intellectualarchive.com/?link $=$ item\&id $=1872$

Hodge, J.C., 2018a, Magnetostatics relation to gravity with experiment that rejects Biot-Savart Law, IntellectualArchive, Vol.7, No. 3, p.1-8 http://intellectualarchive.com/?link=item\&id=1945 See video: https://www.youtube.com/watch?v=A07bogzzMEI

Hodge, J.C., 2018b, Another experiment rejects Ampere's Law and supports the STOE model, IntellectualArchive, Vol.7, No. 4, p.1-5 http://intellectualarchive.com/?link $=$ item\&id $=1956$

Hodge, J.C., 2018c, Two different types of magnetic field, IntellectualArchive, Vol.7, No. 4, p.1-8 http://intellectualarchive.com/?link=item\&id=1964

Hodge, J.C. 2019a. Interference Experiment with a transparent Mask Rejects Wave Models of Light, Optics and Photonics Journal, v.9(6). DOI: 104236/opj.2019.96008. http://www.scrip.org/journal/paperinformation.aspx?paperid=93056. See video: https://www.youtube.com/watch?v=A07bogzzMEI

Hodge, J. C. 2019b. STOE explaination for the "ether wind". IntellectualArchive, v.8(2), P. 15. http://intellectualarchive.com/?link $=$ item\&id $=2111$.

Hodge, J.C. 2019i. Magnetic field evolves to gravity field:4 Atomic structure. IntellectualArchive, v.8(4), $\quad$ P. 30. http://intellectualarchive.com/?link=item\&id=2199.

Hodge, J. C. 2019j. Magnetic field evolves to gravity field:5 Final. IntellectualArchive, v. 8(4), $\quad$ P. 37. http://intellectualarchive.com/?link=item\&id=2233.

Hodge, J.C., 2020, Scalar Theory of Everything (STOE) unites the big, the small, and the four forces (GUT) by extending Newton's model, IntellectualArchive, Vol.9, No. 4, p. 14 http://intellectualarchive.com/?link=item\&id $=2414$

DOI: 10.32370/IA_2020_12_3

Michelson, A.A. \& Morley, E.W., 1987, On the Relative Motion of the Earth and the Luminiforus Ether, American Journal of Science, 34 (203) p. 333-345. 


\section{REFERENCES}

Rautio, J.A., 2020, Variability of decay rates points to a new theory of particles and gravity, https://www.researchgate.net/publication/350314443 and references therein. See also papers by Ephraim Fishbach and his team.

von Flandern, T., 1998. Physics Letters A 250, 1.

Verdad, F., 2019, Diffraction and the speed of light, DOI: $10.13140 /$ RG.2.2.25062.98888 